\title{
ARTICLE \\ Diffusion imaging markers of bipolar versus general psychopathology risk in youth at-risk
}

\author{
A Versace ${ }^{1}$, CD Ladouceur ${ }^{1}$, S Graur ${ }^{1}$, HE Acuff ${ }^{2,3}$, LK Bonar ${ }^{1}$, K Monk ${ }^{1}$, A McCaffrey ${ }^{1}$, A Yendiki ${ }^{4}$, A Leemans ${ }^{5}$, MJ Travis ${ }^{6}$, VA Diwadkar ${ }^{6}$,
} SK Holland ${ }^{6}$, JL Sunshine ${ }^{6}$, RA Kowatch ${ }^{6}$, SM Horwitz ${ }^{6}$, TW Frazier ${ }^{6}$, LE Arnold ${ }^{8}$, MA Fristad ${ }^{6}$, EA Youngstrom ${ }^{6}$, RL Findling ${ }^{6}$, BI Goldstein ${ }^{7}$, T Goldstein $^{1}$, D Axelson ${ }^{8}$, B Birmaher ${ }^{1}$ and ML Phillips ${ }^{1}$

Bipolar disorder (BD) is highly heritable. Thus, studies in first-degree relatives of individuals with BD could lead to the discovery of objective risk markers of BD. Abnormalities in white matter structure reported in at-risk individuals could play an important role in the pathophysiology of BD. Due to the lack of studies with other at-risk offspring, however, it remains unclear whether such abnormalities reflect BD-specific or generic risk markers for future psychopathology. Using a tract-profile approach, we examined 18 major white matter tracts in 38 offspring of BD parents, 36 offspring of comparison parents with non-BD psychopathology (depression, attention-deficit/hyperactivity disorder), and 41 offspring of healthy parents. Both at-risk groups showed significantly lower fractional anisotropy (FA) in left-sided tracts (cingulum, inferior longitudinal fasciculus, forceps minor), and significantly greater FA in right-sided tracts (uncinate fasciculus and inferior longitudinal fasciculus), relative to offspring of healthy parents $(P<$ 0.05). These abnormalities were present in both healthy and affected youth in at-risk groups. Only offspring (particularly healthy offspring) of BD parents showed lower FA in the right superior longitudinal fasciculus relative to healthy offspring of healthy parents $(P<0.05)$. We show, for the first time, important similarities, and some differences, in white matter structure between offspring of $B D$ and offspring of non-BD parents. Findings suggest that lower left-sided and higher right-sided FA in tracts important for emotional regulation may represent markers of risk for general, rather than BD-specific, psychopathology. Lower FA in the right superior longitudinal fasciculus may protect against development of BD in offspring of BD parents.

Neuropsychopharmacology (2018) 43:2212-2220; https://doi.org/10.1038/s41386-018-0083-z

\section{INTRODUCTION}

Bipolar disorder (BD) is highly heritable and a leading cause of morbidity worldwide [1]. The identification of risk markers is critical to improve early diagnosis. First-degree relatives, including offspring of bipolar parents (OBP), are at higher risk for mood disorders, than offspring of healthy parents (OHP), and offspring of comparison parents (OCP) with non-BD psychopathology (e.g., depression, attention-deficit/hyperactivity disorder) [2-5]. Several factors could explain the variation in reported rates of $\mathrm{BD}$ onset in studies of BD offspring, including differences in age range, sample size, diagnostic criteria, and study design (i.e., longitudinal vs retrospective self-reports). Longitudinal studies in $\mathrm{OBP}$ indicate that children with two parents with $\mathrm{BD}$ are at higher risk (up to $30 \%$ ) for $\mathrm{BD}$ than those having one parent with $\mathrm{BD}$ (10.6-19.2\%) and OHP (0.6-2\%) [2-5]. One study, using retrospective self-reports in $1861 \mathrm{OCP}$, reported estimates of BD onset in $12 \%$ of OCP before age 25 [6]. Abnormalities in neural functioning and structure most likely mediate the relationship between familial risk and onset of $\mathrm{BD}$, but it remains unclear which pathophysiologic mechanisms predispose to or protect against the illness onset.

Neuroimaging studies in BD report functional abnormalities across emotion processing, emotion regulation, and reward circuitries [7]. Lower fractional anisotropy(FA), reflecting lower fiber collinearity, has been consistently reported in white matter tracts connecting prefrontal and subcortical regions in $B D$, and may represent the structural basis for emotional dysregulation in $\mathrm{BD}$ [7]. A systematic review of 37 neuroimaging studies in 966 individuals at-risk for BD (and 1258 controls), highlighted greater activity in predominantly left-sided cortical and insula regions implicated in emotional regulation in BD atrisk vs healthy individuals, independent of fMRI task [8]; but there were no significant between-group differences in gray and white matter fronto-temporal regions [8]. Using voxelbased-morphometry (VBM, including tract-based spatial statistics and wholebrain voxel-based morphometry), subsequent studies in BD at-risk individuals reported abnormalities (i.e., reduced [9] and increased [10] FA) in widespread white matter regions. Nonetheless, these findings were not replicated in a

\footnotetext{
${ }^{1}$ Department of Psychiatry, Western Psychiatric Institute and Clinic, University of Pittsburgh Medical Center, University of Pittsburgh, Pittsburgh, PA, USA; ${ }^{2}$ Departments of

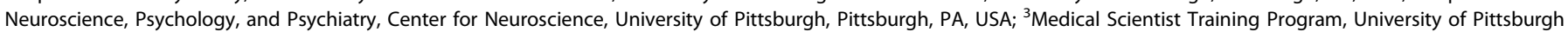

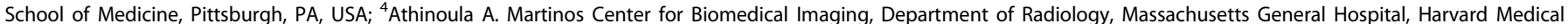

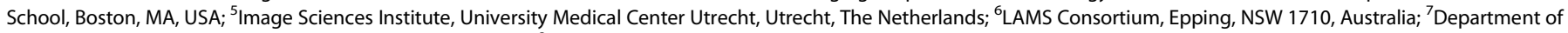

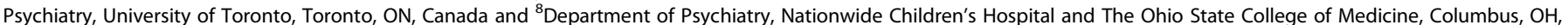
USA

Correspondence: A Versace (versacea@upmc.edu)
}

Received: 15 December 2017 Revised: 31 March 2018 Accepted: 10 April 2018

Published online: 4 May 2018 
subsequent tractography study [11]. Methodological factors, such as sampling differences, use of different neuroimaging protocols/paradigms, rigor of quality control procedures, and power issues are likely to play a role in the discrepancies between studies [8].

Given that OBP and OCP are likely to have a parent with non-BD psychopathology (parents with BD often have comorbid non-BD disorders [12], and both groups experience living with an affected relative, both groups are at familial risk for psychopathology in general. Therefore, neural abnormalities in OBP may represent markers of generic risk for psychopathology rather than markers of $\mathrm{BD}$ risk. Additionally, non-BD psychopathology is a risk factor for $\mathrm{BD}$ in $\mathrm{OBP}$, whereas absence of non-BD psychopathology may represent a protective factor $[2,7]$. Thus, while familial risk for $B D$ is greater in OBP than OCP, as highlighted above, OBP with non$B D$ psychopathology may be at greatest risk of $B D$, with the risk lower in OBP without non-BD psychopathology. Neuroimaging studies comparing OBP and OCP are thus needed to parse markers of specific risk for BD from those for psychopathology in general. To our knowledge, only one diffusion imaging study included OBP and OCP, but the small sample $(n=7)$ precluded group comparison [13]. Furthermore, identifying differential patterns of white matter abnormalities in OBP vs OCP (relative to $\mathrm{OHP}$ ) as a function of non-BD psychopathology can help identify risk vs protective markers of BD by comparing affected vs healthy OBP, and help identify risk vs protective markers of psychopathology in general by comparing affected vs healthy OBP and OCP.

The bipolar offspring study (BIOS) is an ongoing longitudinal study examining the development of psychiatric symptomatology in OBP and OCP. As highlighted above, the latter group controls for presence of non-BD psychopathology in the parent and environmental factors associated with living with a parent with psychiatric illness[14]. The goal of the present neuroimaging study was to: (1) identify diffusion imaging measures associated with risk for $\mathrm{BD}$ vs risk for psychopathology in general, by comparing OBP, $\mathrm{OCP}$, and OHP; (2) explore the extent to which these measures are associated with risk for, vs protection against, BD and non-BD psychopathology, by comparing affected and healthy OBP (or OCP) vs OHP.

Use of tract-profile rather than conventional measures (i.e., mean of diffusion imaging properties across the entire tract), allowed us to examine the extent to which putative markers of risk for BD or psychopathology in general were widespread or localized in focal portions (nodes) of a given tract. No previous studies employed such an approach in the study of BD risk.

We hypothesized that:

i. Relative to OHP, white matter abnormalities exhibited by all (affected and healthy) OBP and OCP would represent risk markers of psychopathology in general, whereas white matter abnormalities exhibited by all OBP, but not OCP, would represent specific risk markers of $\mathrm{BD}$. Given the inconsistent findings in $\mathrm{OBP}$, we based our hypotheses on findings in individuals with $\mathrm{BD}[2,7]$. We predicted that white matter abnormalities would be evident in tracts connecting prefrontal and subcortical regions, including forceps minor, cingulum, uncinate fasciculus and the anterior limb of the internal capsule. As no studies directly compared OCP, OBP, and OHP, we could not hypothesize which tracts would distinguish OBP from OCP.

ii. Relative to OHP, white matter abnormalities in healthy, but not affected, OBP and OCP would represent potential protective markers against psychopathology, whereas white matter abnormalities in healthy OBP, but not other at-risk groups (healthy OCP, affected $\mathrm{OCP}$, and affected $\mathrm{OBP}$ ) would represent potential protective markers against $\mathrm{BD}$. The absence of studies of this nature prevented us from hypothesizing which specific tracts would show such abnormalities.

\section{METHODS}

Participants

Seventy-four youth (7-17 years) with no current/history of BD were recruited from BIOS [14]: OBP $(n=38)$ and OCP $(n=36)$. Forty-one healthy OHP matched with OBP and OCP on age, gender, handedness, IQ, and education level were recruited from the Longitudinal Assessment of Manic Symptoms (LAMS) study [15], Pittsburgh site. Exclusion criteria are in Supplemental Materials. $3 \mathrm{OBP}, 3 \mathrm{OCP}$, and $2 \mathrm{OHP}$ were excluded from analysis due to missing data (2 OBP, 2 OHP) or image artifacts (1 OBP, 3 OCP; eTable-1A). Those excluded did not differ significantly from those included on age, sex, or IQ, leaving 107 useable scans in 35 OBP, 33 OCP and 39 OHP participants (Table 1). Of these, 20 OBP and 19 OCP had no current psychopathology, while 15 OBP and 14 OCP were affected by two or more comorbid diagnoses at scan (Table 1).

Symptom assessment. Parental psychopathology was ascertained by a trained clinician using the Structural Clinical Interview for DSM-IV (SCID-I) [16] for BIOS youth, and using detailed clinical assessment for LAMS youth. Another trained clinician, blind to parental diagnosis, interviewed parents about their children, and interviewed their children, using the Kiddie Schedule for Affective Disorders and Schizophrenia (KSADS) [17]. All cases were supervised by a child psychiatrist, who was responsible for determining final diagnoses. Inter-rater reliability for the KSADS was $>0.8$. On the scan day, youth and their parents/guardians completed the child/parent versions of the: Self-Report for Childhood Anxiety Related Emotional Disorders(SCARED-C/P) [18], Children's Affective Lability Scale(CALS-C/P) [19], and Mood and Feelings Questionnaire(MFQ-C/P) [20] to assess symptom severity during the 2 weeks preceding the scan. KSADS Mania Rating Scale(K-MRS), Depression Rating Scale(K-DRS) [21], and Child/Adolescent Symptom Inventory [22] assessed hypo/manic, depressive, and ADHD symptoms up to 6 months preceding the scan.

Data analysis

Neuroimaging. Protocol and preprocessing steps are described in Supplemental Materials. Analysis of 18 major white matter tract tractograms was performed using ExploreDTI (www.exploredti. com) and TRACULA (www.freesurfer.net/fswiki/Tracula) in the FreeSurfer package. The tensor model was fitted to the data to extract FA and other tensor-based measures in each participant. FA represents the degree of fiber coherence (collinearity). Axial and radial diffusivity $(A D, R D)$, which represent water displacement along principal and non-principal diffusion directions, can help interpret FA abnormalities [23]. These measures were extracted from tract-profiles of major white matter tracts using the global probabilistic approach [24]. (Supplemental Materials).

Statistical approach. Demographic, clinical, and diffusion imaging measures were imported into SPSS (version 24) to test main hypotheses. Given effects of age (linear and quadratic effects) [25, 26] and sex [27] on white matter, these variables were covariates in all analyses.

To test our hypotheses, we adopted the following analytic approach.

Level-1 analyses: Analyses of covariance (ANCOVA) examined the main effect of group (OBP, OCP, and OHP) on FA in each node.

Level-2 analyses: Post hoc ANCOVAs in OBP (or OCP) vs OHP and in OBP vS OCP determined the nature of between-group differences in FA in each node showing a main effect of group in Level-1 analyses. 
Table-1. Demographic and clinical variables in $35 \mathrm{OBP}, 33 \mathrm{OCP}$, and $39 \mathrm{OHP}$

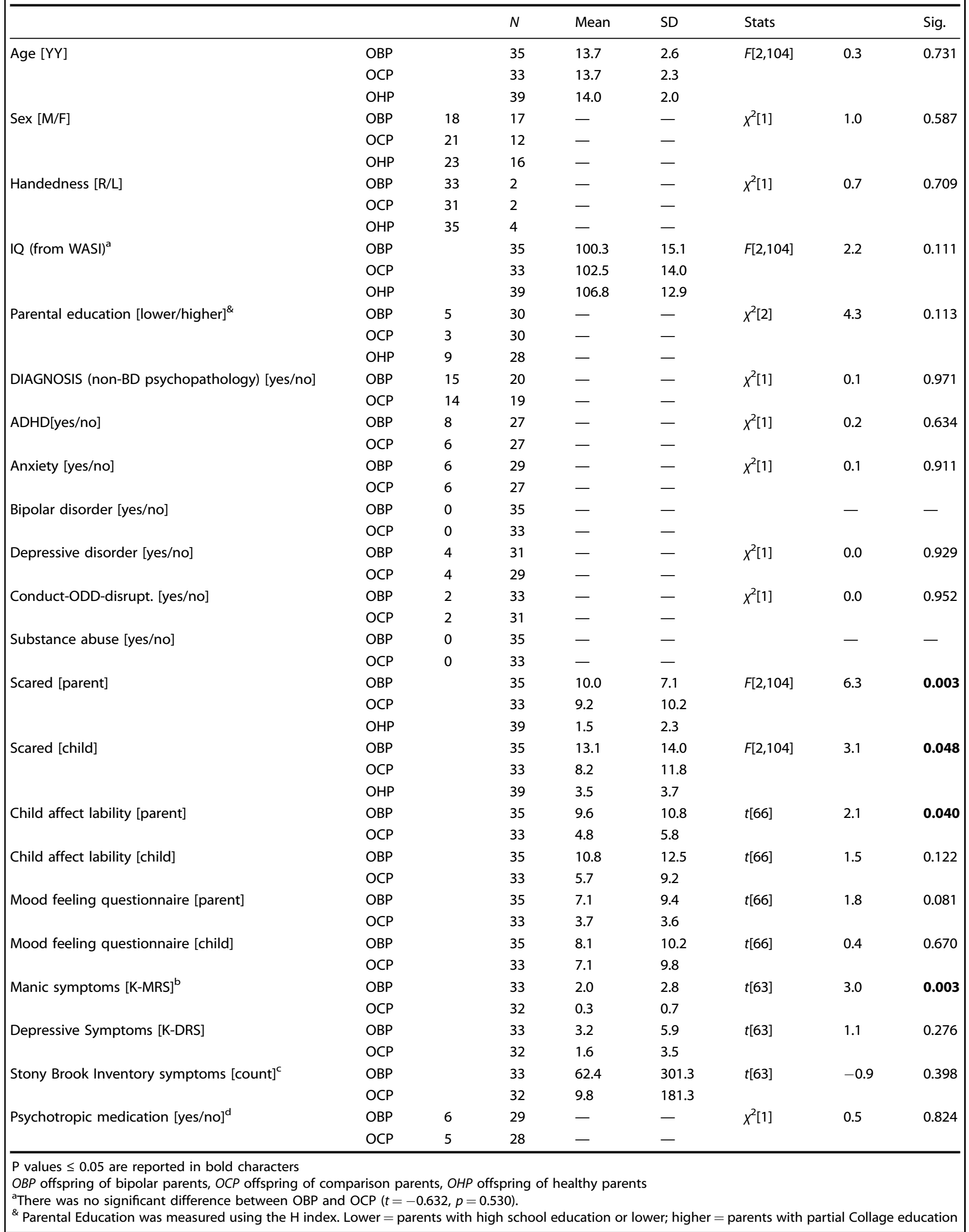


or higher

Missing information on parental education in 2 OHP.

${ }^{\mathrm{b}}$ Affected OBP reported higher levels of subthreshold manic symptoms than healthy OBP $\left(t_{[23]}=3.2, p=0.006\right)$; healthy OCP $\left(t_{[23]}=3.7, p=0.002\right)$ and affected $\operatorname{OCP}\left(t_{[23]}=3.5, p=0.002\right)$

'Symptom Count is based on the number of symptoms necessary for a DSM-IV diagnosis

${ }^{\mathrm{d}}$ All participants completed medication forms that documented psychotropic medications used at the time of the scan. Five OBP (antipsychotics: $n=2$, nonstimulants $=2$ and stimulants: $n=1$ ) and six OCP (antidepressants: $n=3$ and stimulants: $n=3$ ) were taking one class of psychotropic medications, and one OBP was taking a combination (antipsychotic, topiramate and antidepressant) therapy

Level-3 analyses: Given the small number of offspring with a (non-BD) diagnosis (Table 1), we did not include these variables as independent factors in models in level 1 and 2 analyses. Level-3 analyses explored the effect of non-BD psychopathology in OBP (or OCP), by comparing affected OBP (or OCP) vs OHP on FA in each node showing between-group differences in level-2 analyses. Parallel analyses were performed in healthy OBP (or OCP) vs OHP. Very few offspring were medicated (Table 1), therefore, we did not examine the impact of medication on main findings.

To account for multiple comparisons (all nodes), a false discovery rate (FDR) correction was used $(p<0.05)$ in all analyses. Specifically, for each model (level 1-3) all nodal uncorrected $p$ values were imported into R-studio and an FDR correction was applied using the p.adjust function.

Between-group differences in mean $A D$ and RD were examined in each cluster-region (i.e., diffusivity measures were averaged across all contiguous nodes within the same anatomical region of a given tract) showing a main effect of group on FA, paralleling level 2-3 analyses.

Exploratory analysis. In $\mathrm{OBP}$ and $\mathrm{OCP}$, we explored relationships between symptom severity (SCARED-C/P, CALS-C/P, MFQ-C/P, KMRS, K-DRS, Child/Adolescent Symptom Inventory) as rated by OBP and OCP and their parents and FA in each cluster-region showing a main effect of group in level-1 analyses, using Pearson's correlations with FDR correction for multiple comparisons. The main effect of non-BD psychopathology (yes/no) was also explored, using a multivariate multiple regression analysis.

\section{RESULTS}

Demographic and clinical characteristics

There were no significant between-group differences in age, sex ratio, handedness, SES, and IQ. OBP and OCP had significantly more anxiety (SCARED), mood lability (CALS) and affective (MFQ) symptoms than OHP. OBP reported higher levels of manic symptoms than OCP (Table 1).

Diffusion imaging

All reported findings are FDR corrected for multiple comparisons.

Level-1 analyses. After accounting for age (linear and quadratic effects) and sex, multiple (one for each node) ANCOVAs revealed a significant effect of group on FA in the medial section of the forceps minor, in the left hemisphere $\left(F_{[2,95]}=7.6 ; P=0.020\right)$, in the left cingulum bundle $\left(F_{[2,95]}=16.7 ; P=0.009\right)$, the middle portion of the left inferior longitudinal fasciculus $\left(F_{[2,95]}=8.6 ; P=\right.$ $0.024)$, the middle portion of the right inferior longitudinal fasciculus $\left(F_{[2,95]}=8.2 ; P=0.012\right)$, the anterior portion of the right uncinate fasciculus, near the anterior temporal pole $\left(F_{[2,95]}=\right.$ 9.0; $\mathrm{P}=0.004)$, and the middle portion of the right superior longitudinal fasciculus $\left(F_{[2,95]}=8.6 ; P<0.001\right)$ (Fig. 1). There was a trend for a quadratic (inverted $U$ ) effect of age in the left anterior thalamic radiation $(P<0.050)$, right superior longitudinal fasciculus $(P<0.040)$, and inferior longitudinal fasciculus (left: $P<0.045$; right: $P<0.047)$, but these effects did not survive FDR correction for multiple comparisons.
Level-2 analyses. After accounting for age and sex, OBP and OCP showed lower FA than OHP in the medial section of the forceps minor, in the left hemisphere (OBP-vs-OHP: $F_{[1,61]}=6.8 ; P=0.030$; OCP-vs-OHP: $\left.F_{[1,67]}=13.6 ; P=0.002\right)$, the left cingulum bundle (OBP-vs-OHP: $F_{[1,61]}=16.7 ; P=0.011$; OCP-vs-OHP: $F_{[1,67]}=29.3 ; P$ $=0.001)$, and the middle portion of the left inferior longitudinal fasciculus (OBP-vs-OHP: $F_{[1,61]}=11.5 ; \quad P=0.004$; OCP-vs-OHP: $F_{[1,67]}=7.9 ; P=0.020$; Fig. 1a, Tables 2, and 3). OBP, but not $\mathrm{OCP}$, showed lower FA in the middle portion of the right superior longitudinal fasciculus (OBP-vs-OHP: $F_{[1,61]}=8.9 ; P=0.008$ ).

By contrast, both OBP and OCP showed higher FA than OHP in the middle portion of the right inferior longitudinal fasciculus (OBP-vs-OHP: $F_{[1,61]}=9.6 ; P=0.007 ;$ OCP-vs-OHP: $F_{[1,67]}=14.0 ; P$ $=0.003)$ and the anterior portion of the right uncinate fasciculus (OBP-vs-OHP: $F_{[1,61]}=9.8 ; P=0.008$; OCP-vs-OHP: $F_{[1,67]}=0.6 ; P=$ 0.005 ) (Fig. 1b, Tables 2, and 3).

There was no significant difference in FA in any of the above nodes in OBP vs OCP.

Level-3 analyses. Healthy OBP and OCP showed lower FA than $\mathrm{OHP}$ in the medial portion of the forceps minor, in the left hemisphere (OBP-vs-OHP: $F_{[1,48]}=5.6 ; P=0.049$; OCP-vs-OHP: $F_{[1,50]}=6.9 ; P=0.038$ ), and the left cingulum bundle (OBP-vsOHP: $F_{[1,48]}=9.7 ; P=0.035$; OCP-vs-OHP: $\left.F_{[1,50]}=20.0 ; P=0.002\right)$. OBP, but not OCP, showed lower FA than OHP in the left inferior longitudinal fasciculus (OBP-vs-OHP: $F_{[1,48]}=6.0 ; P=0.046$ ) and the middle portion of the right superior longitudinal fasciculus (OBP-vs-OHP: $F_{[1,61]}=7.0 ; P=0.034$ ).

Healthy OBP and healthy OCP showed higher FA than OHP in the middle portion of the right inferior longitudinal fasciculus (OBP-vs-OHP: $F_{[1,48]}=4.2 ; P=0.050 ;$ OCP-vs-OHP: $F_{[1,50]}=13.7 ; P$ $=0.014)$ and in the anterior portion of the right uncinate fasciculus (OBP-vs-OHP: $F_{[1,48]}=7.2 ; \quad P=0.034 ; \quad$ OCP-vs-OHP: $\left.F_{[1,50]}=8.4 ; P=0.022\right)$.

There were no significant differences in FA in any of the above nodes in healthy OBP vs healthy OCP.

Affected OCP, but not OBP, showed lower FA than OHP in the medial portion of the forceps minor in the left hemisphere (OCPvs-OHP: $\left.F_{[1,45]}=10.4 ; P=0.011\right)$. OBP and OCP showed lower FA in

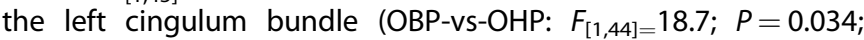
OCP-vs-OHP, $\left.F_{[1,45]}=14.4 ; P=0.008\right)$ and the middle portion of the left inferior longitudinal fasciculus (OBP-vs-OHP; $F_{[1,44]}=8.1 ; P$ $=0.021 ;$ OCP-vs-OHP, $\left.F_{[1,45]}=9.2 ; P=0.015\right)$. Affected OBP did not show any difference in FA relative to OHP in the right superior longitudinal fasciculus.

Affected OBP and affected OCP showed higher FA than OHP in the middle portion of the right inferior longitudinal fasciculus (OBP-vs-OHP; $F_{[1,44]}=12.2 ; P=0.016 ;$ OCP-vs-OHP; $F_{[1,45]}=6.5 ; P$ $=0.035)$ and the anterior portion of the right uncinate fasciculus (OCP-vs-OHP; $F_{[1,45]}=6.4 ; P=0.038 ;$ OBP-vs-OHP showed lower FA in this cluster; however, this finding was no longer significant after FDR correction).

There were no significant differences in FA in any of the above nodes in affected OBP vs affected OCP.

$A D$ and $R D$ findings are in Supplemental Materials.

Exploratory analysis. There were no significant relationships between symptom severity and FA in any cluster-region showing 


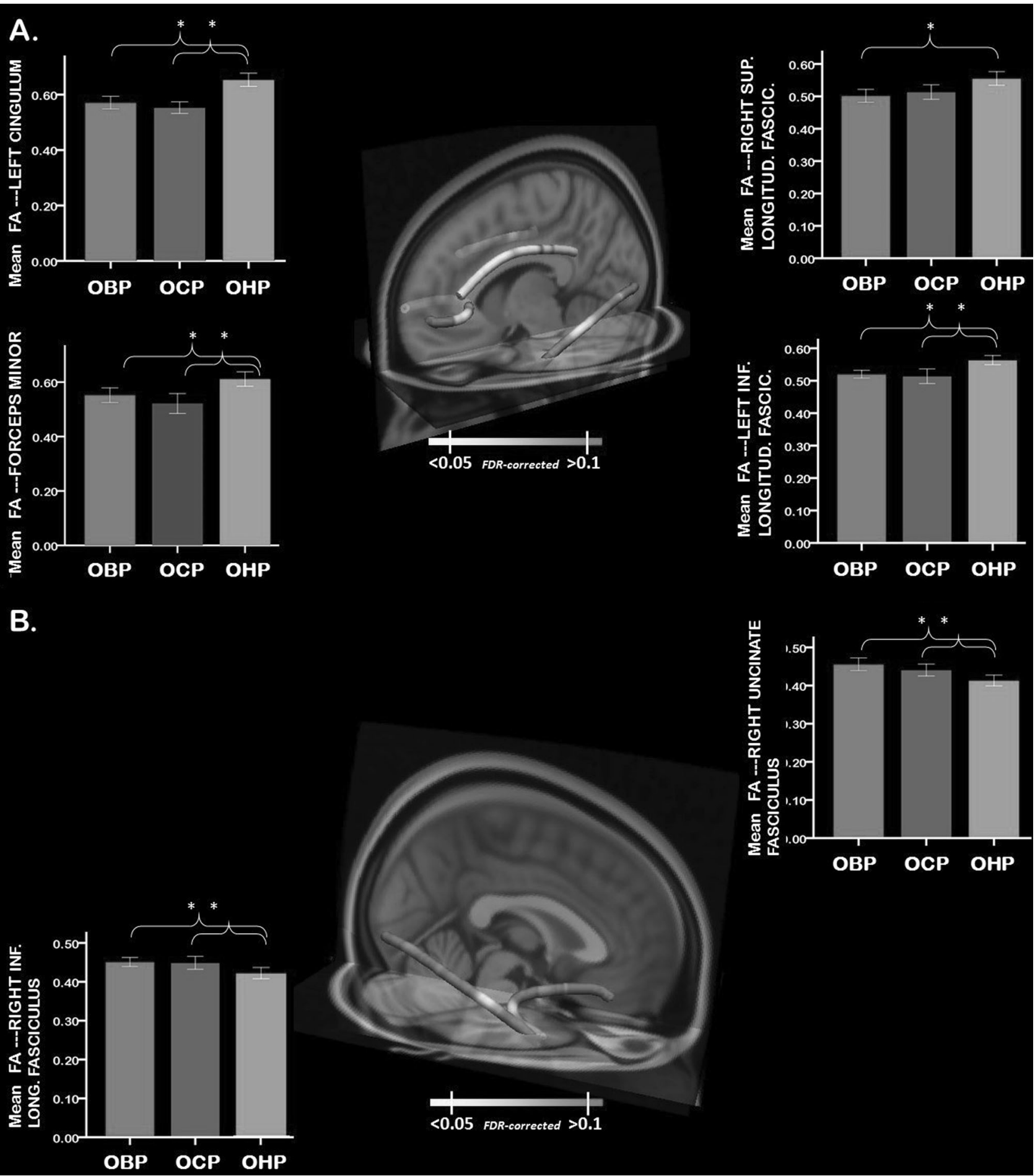

Fig. 1 a 3D visualization of white matter tracts showing lower FA in OBP (and OCP) vs OHP. b 3D visualization of white matter tracts showing greater FA in OBP (and OCP) vs OHP. Error bar plots represent the mean FA (averaged FA across nodes) in white matter tracts with a main effect of group (FDR-corrected) in OBP-vs-OHP. Tractographic analyses were performed in native space (eFigure-1). Nodal measures along each tract were derived in native space for each subject using the dmri_pathstats function. This approach allowed us to define tract-profiles for each white matter tract of interest in each subject. To combine these tract-profiles for group-level analyses, nodal diffusivity measures were interpolated at corresponding positions along any given tract, using the trac-all -stat function, as proposed in https://surfer.nmr.mgh.harvard. edu/fswiki /FsTutorial/TraculaStatistics across all subjects. Specifically, tract-profile analysis yielded $N=53$ nodes which were common across all subjects in the forceps minor, $N=35$ in the left/right anterior thalamic radiation, $N=30$ in the left/right cingulum, $N=38$ in the left/right inferior longitudinal fasciculus, $N=50$ in the left/right arcuate; $N=38$ in the left/right superior longitudinal fasciculus; $N=29$ in the left/right uncinate fasciculus; $N=58$ in the cortico-spinal tract. Nodal diffusivity measures were then extracted and imported in SPSS for level $1-3$ analyses, as described in the Methods section. For display purposes, findings (i.e., nodal FDR-corrected $p$-values) were then saved in text files (one for each analysis) and uploaded in MNI space to represent between group-differences, using freeview (graphical toolbox of TRACULA; asterisks show between group differences). OBP offspring of bipolar parents; OCP offspring of comparison parents; OHP offspring of healthy parents 


\begin{tabular}{|c|c|}
\hline Comparison $^{\mathrm{a}}$ & $\mathrm{FA}(\downarrow \uparrow)$ \\
\hline \multicolumn{2}{|l|}{ Forceps minor } \\
\hline OBP-vs-OHP & $\downarrow$ \\
\hline OCP-vs-OHP & $\downarrow$ \\
\hline OBP-vs-OCP & $\cong$ \\
\hline \multicolumn{2}{|c|}{ Left cingulum bundle } \\
\hline OBP-vs-OHP & $\downarrow$ \\
\hline OCP-vs-OHP & $\downarrow$ \\
\hline OBP-vs-OCP & $\cong$ \\
\hline \multicolumn{2}{|c|}{ Left inferior longitudinal fasciculus } \\
\hline OBP-vs-OHP & $\downarrow$ \\
\hline OCP-vs-OHP & $\downarrow$ \\
\hline OBP-vs-OCP & $\cong$ \\
\hline \multicolumn{2}{|c|}{ Right superior longitudinal fasciculus } \\
\hline OBP-vs-OHP & $\downarrow$ \\
\hline OCP-vs-OHP & $\cong$ \\
\hline OBP-vs-OCP & $\cong$ \\
\hline \multicolumn{2}{|c|}{ Right inferior longitudinal fasciculus } \\
\hline OBP-vs-OHP & $\uparrow$ \\
\hline OCP-vs-OHP & $\uparrow$ \\
\hline OBP-vs-OCP & $\cong$ \\
\hline \multicolumn{2}{|c|}{ Right uncinate fasciculus } \\
\hline OBP-vs-OHP & $\uparrow$ \\
\hline OCP-vs-OHP & $\uparrow$ \\
\hline OBP-vs-OCP & $\cong$ \\
\hline
\end{tabular}

an effect of group in level-1 analyses (eTable-2; all FDR-corrected $P>0.05$ ). Exclusion of parental reports from analyses did not yield any significant findings (eTable-2 footnote). Similarly, there was no effect of non-BD psychopathology (eTable-2).

\section{DISCUSSION}

The goal of the present study was to: (1) identify abnormalities in diffusivity measures of white matter tracts implicated in emotional regulation in youth at-risk for BD vs youth at-risk for psychopathology in general; and (2) explore whether these diffusivity measures were associated with risk for, vs protection against, BD and psychopathology in general.

In partial support of our primary hypothesis, our findings demonstrate that both OBP and OCP show abnormal collinearity in white matter tracts implicated in emotional regulation: lower FA in predominantly left-sided tracts and higher FA in predominantly right-sided tracts. Specifically, relative to OHP, they showed lower $F A$ in the middle portion of the forceps minor and left cingulum and greater FA in right uncinate fasciculus. Additionally, both OBP and OCP vs OHP showed lower FA in the middle portion of the left inferior longitudinal fasciculus and higher FA in the right inferior longitudinal fasciculus.

FA abnormalities in left-sided white matter tracts implicated in emotional regulation are reported in individuals with different psychiatric disorders. Lower FA in left-sided tracts was reported in a meta-analysis of 37 VBM studies in individuals with different psychiatric disorders $(n=962)$, including major depressive disorder, BD, social anxiety, obsessive-compulsive disorder and posttraumatic stress disorder [28]. Relative to healthy controls,
Table 3. White matter tracts showing significantly lower FA in OBP and/or OCP vs OHP

\begin{tabular}{|c|c|c|c|}
\hline & $k$ & $F$ & $\begin{array}{l}P \text {-value (FDR } \\
\text { corrected) }\end{array}$ \\
\hline \multicolumn{4}{|l|}{ Forceps minor ${ }^{a}$} \\
\hline Level-1 analyses (OBP, OCP, OHP) & 7 & 7.6 & 0.020 \\
\hline Level-2 analyses (OBP, OHP) & 6 & 6.8 & 0.030 \\
\hline Level-2 analyses (OCP, OHP) & 7 & 13.6 & 0.002 \\
\hline Level-3 analyses (healthy OBP, OHP) & 4 & 5.6 & 0.049 \\
\hline Level-3 analyses (healthy OCP, OHP) & 7 & 6.9 & 0.038 \\
\hline Level-3 analyses (affected OBP, OHP) & - & - & - \\
\hline Level-3 analyses (affected OCP, OHP) & 7 & 10.4 & 0.011 \\
\hline \multicolumn{4}{|l|}{ Left cingulum bundle ${ }^{a}$} \\
\hline Level-1 analyses (OBP, OCP, OHP) & 28 & 16.7 & 0.009 \\
\hline Level-2 analyses (OBP, OHP) & 26 & 16.7 & 0.011 \\
\hline Level-2 analyses (OCP, OHP) & 28 & 29.3 & 0.001 \\
\hline Level-3 analyses (healthy OBP, OHP) & 25 & 9.7 & 0.035 \\
\hline Level-3 analyses (healthy OCP, OHP) & 28 & 20.0 & 0.002 \\
\hline Level-3 analyses (affected OBP, OHP) & 16 & 18.7 & 0.034 \\
\hline Level-3 analyses (affected OCP, OHP) & 27 & 14.4 & 0.008 \\
\hline \multicolumn{4}{|l|}{ Left inferior longitudinal fasciculus ${ }^{b}$} \\
\hline Level-1 analyses (OBP, OCP, OHP) & 8 & 8.6 & 0.024 \\
\hline Level-2 analyses (OBP, OHP) & 8 & 11.5 & 0.004 \\
\hline Level-2 analyses (OCP, OHP) & 7 & 7.9 & 0.02 \\
\hline Level-3 analyses (healthy OBP, OHP) & 3 & 6.0 & 0.046 \\
\hline Level-3 analyses (healthy OCP, OHP) & - & - & - \\
\hline Level-3 analyses (affected OBP, OHP) & 7 & 8.1 & 0.021 \\
\hline Level-3 analyses (affected OCP, OHP) & 5 & 9.2 & 0.015 \\
\hline \multicolumn{4}{|l|}{ Right superior longitudinal fasciculus } \\
\hline Level-1 analyses (OBP, OCP, OHP) & 2 & 8.6 & $<0.001$ \\
\hline Level-2 analyses (OBP, OHP) & 2 & 8.9 & 0.008 \\
\hline Level-2 analyses (OCP, OHP) & - & - & - \\
\hline Level-3 analyses (healthy OBP, OHP) & 2 & 7.0 & 0.034 \\
\hline Level-3 analyses (affected OBP, OHP) & - & - & - \\
\hline
\end{tabular}

White matter tracts showing significant greater FA in OBP and/or OCP vs OHP

$\begin{array}{lllll}\text { Right inferior longitudinal fasciculus } & & & \\ \text { Level-1 analyses (OBP, OCP, OHP) } & 6 & 8.2 & 0.012 \\ \text { Level-2 analyses (OBP, OHP) } & 6 & 9.6 & 0.007 \\ \text { Level-2 analyses (OCP, OHP) } & 6 & 14 & 0.003 \\ \text { Level-3 analyses (healthy OBP, OHP) } & 3 & 4.2 & 0.050 \\ \text { Level-3 analyses (healthy OCP, OHP) } & 6 & 13.7 & 0.014 \\ \text { Level-3 analyses (affected OBP, OHP) } & 5 & 12.2 & 0.016 \\ \text { Level-3 analyses (affected OCP, OHP) } & 2 & 6.5 & 0.035 \\ \text { Right uncinate fasciculus } & & & \\ \text { Level-1 analyses (OBP, OCP, OHP) } & 3 & 9 & 0.004 \\ \text { Level-2 analyses (OBP, OHP) } & 3 & 9.8 & 0.008 \\ \text { Level-2 analyses (OCP, OHP) } & 3 & 8.6 & 0.005 \\ \text { Level-3 analyses (healthy OBP, OHP) } & 2 & 7.2 & 0.034 \\ \text { Level-3 analyses (healthy OCP, OHP) } & 2 & 8.4 & 0.022 \\ \text { Level-3 analyses (affected OBP, OHP) } & - & - & - \\ \text { Level-3 analyses (affected OCP, OHP) } & 3 & 6.4 & 0.038\end{array}$

Age, age2, and sex were covariates in all analyses

$O B P$ offspring of bipolar parents, $O C P$ offspring of comparison parents, $O H P$ offspring of healthy parents

'Lower FA was associated with significantly lower $A D$ in healthy $O B P$, affected OBP, healthy OCP, and affected OCP (all FDR corrected $p<0.05$ )

bLower FA was associated with significant lower $A D$ in healthy $O B P$, affected OBP, and healthy OCP 
lower FA was observed across all disorders in the forceps minor (left portion), left superior longitudinal fasciculus, left inferior fronto-occipital fasciculus, and left uncinate fasciculus [28]. Using a different (i.e., tractography) approach, no laterality effect regarding FA abnormalities in similar white matter tracts was shown in youth with emotional and behavioral dysregulation disorders [29]. As in most tractography studies, however, this latter study reported mean FA across the entirety of each tract. Such an approach could reduce the sensitivity to detect subtler abnormalities than the VBM approach [28], and the tract-profile approach used in the present study.

Regardless of the nature of the familial risk and psychopathologic status, all at-risk youth showed lower FA in the left cingulum, relative to OHP. Lower FA in this tract was recently associated with genetic risk for depression in young women with subthreshold symptom severity [30], suggesting that this abnormality might represent a generic marker of having a parent with a psychiatric disorder, regardless of the diagnostic status of the offspring. The cingulum bundle, connecting medial prefrontal with more posterior parieto-temporal cortical regions, is a major tract of the dorso-limbic pathway, primarily involved in emotional processing and reward [31]. Aberrant left-sided activity in this circuitry was reported in individuals with BD [32$36]$, youth with different emotional and behavioral dysregulation disorders [37], and distressed young adults with high-trait sensation seeking [38], suggesting that aberrant connectivity of left-sided circuitry may predispose to emotional and reward processing dysregulation and affective/anxiety-related psychopathology in general.

OBP and OCP also showed lower FA in the middle portion of the left inferior longitudinal fasciculus than OHP. Further analyses revealed that this abnormality was present in healthy and affected OBP and affected OCP. The absence of this abnormality in healthy OCP suggests that lower FA in the left inferior longitudinal fasciculus may represent a specific risk marker for BD as these offspring were potentially the group least at-risk of developing $B D$ after OHP. We reported lower FA in this tract in individuals with major depressive disorder, however [39]. Lower FA in the proximity of the left inferior longitudinal fasciculus, namely inferior fronto-occipital fasciculus, was also reported in a large meta-analysis of VBM studies in adults with emotional disorders [28]. Thus, lower FA in this tract may represent a marker of risk for psychopathology in general. It is possible that the inability to detect lower FA in this tract in healthy OCP reflected insufficient power in level-3 analysis. Future longitudinal studies are needed to clarify the role of this tract in BD vs psychopathology risk in general.

Analyses also revealed greater FA in the middle portion of the right inferior longitudinal fasciculus and, to a lesser extent, in the anterior portion of the right uncinate fasciculus in OBP and OCP relative to OHP. Supplemental analyses revealed an abnormal right > left FA asymmetry in tracts of interest in OBP and OCP (Supplemental Materials). We previously reported greater FA in the right inferior longitudinal fasciculus in a different sample of healthy OBP (relative to OHP). Greater FA in this tract was also reported in another study comparing individuals at-risk for BD relative to healthy individuals $[40,41]$. Other studies documented structural abnormalities in right-sided emotional regulation circuitry in BD [42-45]. Both the uncinate and inferior longitudinal fasciculus are important tracts for emotional processing and regulation [31]. Further studies are needed to determine the role of elevated FA in right-sided emotional regulation tracts in individuals at-risk for $\mathrm{BD}$ and those at-risk for psychopathology in general.

OBP, but not OCP, showed lower FA than OHP in the middle portion of the right superior longitudinal fasciculus, suggesting a tract-specific marker of familial risk for BD. A previous report of lower $\mathrm{FA}$ in this tract in $\mathrm{BD}$ individuals vs their unaffected relatives
[11] further suggests that such abnormalities may also represent a marker of BD psychopathology. Our additional analyses revealed; however, that the abnormal reduction in FA in this tract was significant in healthy OBP, but not in affected OBP. Given that affected OBP are at greater risk for BD than healthy OBP [14] and that OCP did not show this abnormality, it is also possible that lower FA in this tract may represent a protective marker against future BD. Nonetheless, direct comparison of OBP and OCP did not yield any significant differences in FA in any white matter tracts. Further studies are needed to clarify the role of the right superior longitudinal fasciculus in BD.

All at-risk youth (vs OHP) showed lower FA in the medial portion of the forceps minor in the left hemisphere but this finding did not survive stringent multiple comparison correction in affected OBP. Nevertheless, in the context of the observed significantly lower AD in the same region of this tract in all four at-risk groups, relative to $\mathrm{OHP}$, the trend-level finding of lower FA in affected OBP may be a marker of risk for psychopathology in general.

Given the age range of offspring in this study, it is important to consider the current findings from a developmental perspective. Lower FA has been associated with higher RD in adults with mood disorders [46-48], suggestive of abnormal reorganization of axonal architecture and/or myelin damage in these disorders [49]. The association of lower FA and lower AD, but not higher RD, in the forceps minor, left cingulum and left inferior longitudinal fasciculus in all at-risk groups may reflect lower density (i.e., abnormally reduced number) of collinear fibers, rather than abnormal reorganization of the fibers and/or abnormal myelination in these tracts [49]. This pattern of abnormal FA and AD in the above tracts may thus represent a neurodevelopmental marker of predisposition to psychopathology in general, where abnormally low density of collinear fibers in youth may lead to an abnormal compensatory increase of both collinear and non-collinear fibers over time, leading to the patterns of normal $A D$ and higher RD that are reported in adults with mood disorders ([46]). Longitudinal studies are needed to test this hypothesis further.

Certain limitations should be considered. We did not include individual-level genetic risk score in analyses. Larger sample sizes could ensure power in examining the effect of diagnoses, symptom dimensions, and/or medication classes. There was no significant main effect of age or sex, which is inconsistent with studies reporting an inverted $U$ relationship between age and FA in healthy youth and adults $[25,26]$. Although there was a quadratic effect of age in several tracts, this trend did not survive FDR correction. This might due, in part, to the larger proportion of older than younger youth (age was skewed to the right) in our sample. We previously reported a lack of a normative relationship between age and diffusivity measures (FA and RD) in healthy OBP relative to OHP [41]. Thus, it is also possible that the trend quadratic effect of age on FA across all groups in the present study may have been influenced by the lack of a normative age by FA relationship in OBP, and perhaps OCP. Longitudinal neuroimaging studies are needed to properly test the age by FA relationship in $\mathrm{OBP}, \mathrm{OCP}$, and $\mathrm{OHP}$, and can determine relationships among neuroimaging measures and future clinical outcome in OBP and OCP.

This is the first diffusion imaging study to demonstrate important similarities, and some differences, between OBP and OCP. Our findings suggest that lower FA in predominantly leftsided tracts and higher FA in predominantly right-sided tracts implicated in emotional regulation may represent markers of risk for general, rather than BD-specific, psychopathology, in at-risk youth. Conversely, lower FA in the right superior longitudinal fasciculus may represent a protective marker against development of $\mathrm{BD}$ in OBP. Elucidating associations between neural markers and longitudinal clinical course in OBP and OCP will help to shed light on objective risk vs protective markers for BD. 


\section{FUNDING}

The present study was supported by the National Institute of Mental Health grant R01 MH060952-16 (MPI: Drs. Birmaher and Phillips), by the National Institute of Health/ National Center for Advancing Translational Sciences grant TL1 TR001858-01 (Dr. Wishwa Kapoor) and by the National Institute of Mental Health grants funding the LAMS study (2R01 MH73953-06A1, 2R01, MH73816-06A1, 2R01 MH73967-06A1, and 2R01 MH73801-06A1). These funding agencies were not involved in the design or conduct of the study, the collection, management, analysis, or interpretation of the data, or the preparation, review, or approval of the manuscript. We would like to acknowledge the participants and their families for their contributions to this study.

\section{ADDITIONAL INFORMATION}

The online version of this article (https://doi.org/10.1038/s41386-018-0083-z) contains supplementary material, which is available to authorized users.

Competing interests: V.A., L.C.D., A.H.E., L.A., Y.A., T.M.J., D.V.A., H.S.K., S.J.L., K.R.A., A. D., H.S.M., F.M.A., Y.E.A., G.B.I., and P.M.L., and G.S., M.K., B.L.K., and M.A. have no financial interests or potential conflicts of interest. A.L.E. has received research funding from CureMark, Forest, Lilly, and Shire, advisory board honoraria from Biomarin, Novartis, Noven, Otsuka, Roche, Seaside Therapeutics, and Shire, consulting fees from Tris Pharma, and travel support from Noven. F.T.W. has received federal funding or research support from, acted as a consultant to, received travel support from, and/or received a speaker's honorarium from the Simons Foundation, Ingalls Foundation, Forest Laboratories, Ecoeos, IntegraGen, Shire Development, BristolMyers Squibb, National Institutes of Health, and the Brain and Behavior Research Foundation. F.R.L. receives or has received research support, acted as a consultant and/or served on a speaker's bureau for Aevi, Akili, Alcobra, Amerex, American Academy of Child \& Adolescent Psychiatry, American Psychiatric Press, Bracket, Epharma Solutions, Forest, Genentech, Guilford Press, Ironshore, Johns Hopkins University Press, KemPharm, Lundbeck, Merck, NIH, Neurim, Nuvelution, Otsuka, PCORI, Pfizer, Physicians Postgraduate Press, Purdue, Roche, Sage, Shire, Sunovion, Supernus Pharmaceuticals, Syneurx, Teva, Tris, TouchPoint, Validus, and WebMD. A.D. has served as a consultant for Janssen Research and received royalties from UpToDate. T.G. receives royalties from Guilford Press. B.B. receives or will receive royalties for publications from Random House, Inc. (New Hope for Children and Teens with Bipolar Disorder), Lippincott Williams \& Wilkins (Treating Child and Adolescent Depression), and UpToDate. He is employed by the University of Pittsburgh and the University of Pittsburgh Medical Center/Western Psychiatric Institute and Clinic and receives research funding from NIMH.

Publisher's note: Springer Nature remains neutral with regard to jurisdictional claims in published maps and institutional affiliations.

\section{REFERENCES}

1. Merikangas KR, Akiskal HS, Angst J, Greenberg PE, Hirschfeld RM, Petukhova M, et al. Lifetime and 12-month prevalence of bipolar spectrum disorder in the National Comorbidity Survey replication. Arch Gen Psychiatry. 2007;64:543-52.

2. Hafeman DM, Merranko J, Axelson D, Goldstein BI, Goldstein T, Monk K, et al. Toward the definition of a bipolar prodrome: dimensional predictors of bipolar spectrum disorders in at-risk youths. Am J Psychiatry. 2016;173:695-704.

3. Hafeman DM, Merranko J, Goldstein TR, Axelson D, Goldstein BI, Monk K, et al. Assessment of a person-level risk calculator to predict new-onset bipolar spectrum disorder in youth at familial risk. JAMA Psychiatry. 2017;74:841-7.

4. Axelson D, Goldstein B, Goldstein T, Monk K, Yu H, Hickey MB, et al. Diagnostic Precursors to bipolar disorder in offspring of parents with bipolar disorder: a longitudinal study. Am J Psychiatry. 2015;172:638-46.

5. Birmaher B, Axelson D, Goldstein B, Strober M, Gill MK, Hunt J, et al. Four-year longitudinal course of children and adolescents with bipolar spectrum disorders: the Course and Outcome of Bipolar Youth (COBY) study. Am J Psychiatry. 2009a; 166:795-804.

6. Sucksdorff D, Chudal R, Suominen A, Jokiranta E, Brown AS, Sourander A. Bipolar disorder and parental psychopathology. Soc Psychiatry Psychiatr Epidemiol. 2014;49:1973-84.

7. Phillips ML, Swartz HA. A critical appraisal of neuroimaging studies of bipolar disorder: toward a new conceptualization of underlying neural circuitry and roadmap for future research. Am J Psychiatry. 2014;171:829-43.

8. Fusar-Poli $P$, Howes $O$, Bechdolf A, Borgwardt S. Mapping vulnerability to bipolar disorder: a systematic review and meta-analysis of neuroimaging studies. J Psychiatry Neurosci. 2012;37:170-84.

9. Sprooten E, Sussmann JE, Clugston A, Peel A, McKirdy J, Moorhead TWJ, et al. White matter integrity in individuals at high genetic risk of bipolar disorder. Biol Psychiatry. 2011;70:350-6.
10. Roybal DJ, Barnea-Goraly N, Kelley R, Bararpour L, Howe ME, Reiss AL, et al. Widespread white matter tract aberrations in youth with familial risk for bipolar disorder. Psychiatry Res: Neuroimaging. 2015;232:184-92.

11. Sprooten E, Barrett J, McKay DR, Knowles EE, Mathias SR, Winkler AM, et al. A comprehensive tractography study of patients with bipolar disorder and their unaffected siblings. Hum Brain Mapp. 2016;37:3474-85.

12. Chang $K$, Steiner $H$, Ketter T. Studies of offspring of parents with bipolar disorder. Am J Med Genet Part C, Semin Med Genet. 2003;123c:26-35.

13. Frazier JA, Breeze JL, Papadimitriou G, Kennedy DN, Hodge SM, Moore CM, et al. White matter abnormalities in children with and at risk for bipolar disorder. Bipolar Disord. 2007;9:799-809.

14. Birmaher B, Axelson D, Monk K, Kalas C, Goldstein B, Hickey MB, et al. Lifetime psychiatric disorders in school-aged offspring of parents with bipolar disorder: the Pittsburgh Bipolar Offspring study. Arch Gen Psychiatry. 2009b;66:287-96.

15. Findling RL, Youngstrom EA, Fristad MA, Birmaher B, Kowatch RA, Arnold LE, et al. Characteristics of children with elevated symptoms of mania: the Longitudinal Assessment of Manic Symptoms (LAMS) study. J Clin Psychiatry. 2010;71:1664-72.

16. First MB, Gibbon ML, Spitzer RL, Williams JBW, Benjamin L. Structured Clinical Interview for DSM-IV Axis II Personality Disorders (SCID-II). Washington, DC American Psychiatric Press; 1997.

17. Kaufman J, Birmaher B, Brent D, Rao UMA, Flynn C, Moreci P, et al. Schedule for affective disorders and schizophrenia for school-age children-present and lifetime version (K-SADS-PL): initial reliability and validity data. J Am Acad Child Adolesc Psychiatry. 1997:36:980-8.

18. Birmaher B, Brent DA, Chiappetta L, Bridge J, Monga S, Baugher M. Psychometric properties of the Screen for child anxiety related emotional disorders (SCARED): a replication study. J Am Acad Child Adolesc Psychiatry. 1999;38:1230-6.

19. Gerson AC, Gerring JP, Freund L, Joshi PT, Capozzoli J, Brady K, et al. The children's affective lability scale: a psychometric evaluation of reliability. Psychiatry Res. 1996;65:189-98.

20. Angold A, Costello EJ, Pickles A, Winder F, Silver D. The development of a questionnaire for use in epidemiological studies of depression in children and adolescents. London: Medical Research Council Child Psychiatry Unit; 1987.

21. Axelson D, Birmaher BJ, Brent D, Wassick S, Hoover C, Bridge J, et al. A preliminary study of the kiddie schedule for affective disorders and schizophrenia for schoolage children mania rating scale for children and adolescents. J Child Adolesc Psychopharmacol. 2003;13:463-70.

22. Lavigne JV, Cromley T, Sprafkin J, Gadow KD. The child and adolescent symptom inventory-progress monitor: a brief diagnostic and statistical manual of mental disorders, 4(th) edition-referenced parent-report scale for children and adolescents. J Child Adolesc Psychopharmacol. 2009;19:241-52.

23. Jones D, Leemans A. Diffusion Tensor Imaging. In: Modo M, Bulte JWM (eds) Magnetic Resonance Neuroimaging. Humana Press. New York, NY 10013, USA. 2011;711:127-44.

24. Yendiki A, Panneck P, Srinivasan P, Stevens A, Z[]llei L, Augustinack J et al Automated probabilistic reconstruction of white-matter pathways in health and disease using an atlas of the underlying anatomy. Front Neuroinform 2011;5:1-12.

25. Kochunov P, Williamson DE, Lancaster J, Fox P, Cornell J, Blangero J, et al. Fractional anisotropy of water diffusion in cerebral white matter across the lifespan. Neurobiol Aging. 2012;33:9-20.

26. Lebel C, Gee M, Camicioli R, Wieler M, Martin W, Beaulieu C. Diffusion tensor imaging of white matter tract evolution over the lifespan. Neuroimage. 2012;60:340-52.

27. Simmonds DJ, Hallquist MN, Asato M, Luna B. Developmental stages and sex differences of white matter and behavioral development through adolescence: a longitudinal diffusion tensor imaging (DTI) study. Neuroimage. 2014;92:356-68.

28. Jenkins LM, Barba A, Campbell M, Lamar M, Shankman SA, Leow AD, et al. Shared white matter alterations across emotional disorders: a voxel-based meta-analysis of fractional anisotropy. Neuroimage Clin. 2016;12:1022-34.

29. Versace A, Acuff H, Bertocci MA, Bebko G, Almeida JR, Perlman SB, et al. White matter structure in youth with behavioral and emotional dysregulation disorders: a probabilistic tractographic study. JAMA Psychiatry. 2015;72:367-76.

30. Keedwell PA, Chapman R, Christiansen K, Richardson H, Evans J, Jones DK. Cingulum white matter in young women at risk of depression: the effect of family history and anhedonia. Biol Psychiatry. 2012;72:296-302.

31. Catani M, Dawson MS. Chapter 31 - Language Processing, Development and Evolution A2 - Conn. In: Michael P, ed.. Conn's Translational Neuroscience. San Diego: Academic Press; 2017. p. 679-92.

32. Caseras X, Lawrence NS, Murphy K, Wise RG, Phillips ML. Ventral striatum activity in response to reward: differences between bipolar I and II disorders. Am J Psychiatry. 2013;170:533-41.

33. Chase HW, Nusslock R, Almeida JR, Forbes EE, LaBarbara EJ, Phillips ML. Dissociable patterns of abnormal frontal cortical activation during anticipation of an uncertain reward or loss in bipolar versus major depression. Bipolar Disord. 2013;15:839-54. 
34. Linke J, King AV, Rietschel M, Strohmaier J, Hennerici M, Gass A, et al. Increased medial orbitofrontal and amygdala activation: evidence for a systems-level endophenotype of bipolar I disorder. Am J Psychiatry. 2012;169:316-25.

35. Nusslock R, Almeida JR, Forbes EE, Versace A, Frank E, Labarbara EJ, et al. Waiting to win: elevated striatal and orbitofrontal cortical activity during reward anticipation in euthymic bipolar disorder adults. Bipolar Disord. 2012;14:249-60.

36. Bermpohl F, Kahnt T, Dalanay U, Hagele C, Sajonz B, Wegner T, et al. Altered representation of expected value in the orbitofrontal cortex in mania. Hum Brain Mapp. 2010;31:958-69.

37. Bebko G, Bertocci MA, Fournier JC, Hinze AK, Bonar L, Almeida JR, et al. Parsing dimensional vs diagnostic category-related patterns of reward circuitry function in behaviorally and emotionally dysregulated youth in the Longitudinal Assessment of Manic Symptoms study. JAMA Psychiatry. 2014;71:71-80.

38. Chase HW, Fournier JC, Bertocci MA, Greenberg T, Aslam H, Stiffler R, et al. A pathway linking reward circuitry, impulsive sensation-seeking and risky decisionmaking in young adults: identifying neural markers for new interventions. Transl Psychiatry. 2017;7:e1096.

39. Versace A, Almeida JR, Quevedo K, Thompson WK, Terwilliger RA, Hassel S, et al. Right orbitofrontal corticolimbic and left corticocortical white matter connectivity differentiate bipolar and unipolar depression. Biol Psychiatry. 2010a;68:560-7.

40. Chaddock CA, Barker GJ, Marshall N, Schulze K, Hall MH, Fern A, et al. White matter microstructural impairments and genetic liability to familial bipolar I disorder. Br J Psychiatry. 2009;194:527-34.

41. Versace A, Ladouceur CD, Romero S, Birmaher B, Axelson DA, Kupfer DJ, et al. Altered development of white matter in youth at high familial risk for bipolar disorder: a diffusion tensor imaging study. J Am Acad Child Adolesc Psychiatry. 2010b;49:1249-59.e1241.

42. Foland-Ross LC, Thompson PM, Sugar CA, Madsen SK, Shen JK, Penfold C, et al. Investigation of cortical thickness abnormalities in lithium-free adults with bipolar I disorder using cortical pattern matching. Am J Psychiatry. 2011;168:530-9.

43. Hajek T, Cullis J, Novak T, Kopecek M, Blagdon R, Propper L, et al. Brain structural signature of familial predisposition for bipolar disorder: replicable evidence for involvement of the right inferior frontal gyrus. Biol Psychiatry. 2013;73:144-52.

44. Hallahan B, Newell J, Soares JC, Brambilla P, Strakowski SM, Fleck DE, et al. Structural magnetic resonance imaging in bipolar disorder: an international collaborative mega-analysis of individual adult patient data. Biol Psychiatry. 2011;69:326-35.

45. Haller S, Xekardaki A, Delaloye C, Canuto A, Lovblad KO, Gold G, et al. Combined analysis of grey matter voxel-based morphometry and white matter tract-based spatial statistics in late-life bipolar disorder. J Psychiatry Neurosci. 2011;36:391-401.

46. Emsell L, Langan C, Van Hecke W, Barker GJ, Leemans A, Sunaert S et al. White matter differences in euthymic bipolar I disorder: a coombined magnetic resonance imaging and diffusion tensor imaging voxel-based study. Bipolar Disord. 2013;15:365-76

47. Versace A, Almeida JR, Hassel S, Walsh ND, Novelli M, Klein CR, et al. Elevated left and reduced right orbitomedial prefrontal fractional anisotropy in adults with bipolar disorder revealed by tract-based spatial statistics. Arch Gen Psychiatry. 2008;65:1041-52.

48. Benedetti F, Bollettini I, Poletti S, Locatelli C, Lorenzi C, Pirovano A, et al. White matter microstructure in bipolar disorder is influenced by the serotonin transporter gene polymorphism 5-HTTLPR. Genes Brain Behav. 2015;14:238-50.

49. Sun SW, Liang HF, Le TQ, Armstrong RC, Cross AH, Song SK. Differential sensitivity of in vivo and ex vivo diffusion tensor imaging to evolving optic nerve injury in mice with retinal ischemia. Neuroimage. 2006;32:1195-204.

50. Westlye LT, Walhovd KB, Dale AM, Bjornerud A, Due-Tonnessen P, Engvig A, et al. Life-span changes of the human brain white matter: diffusion tensor imaging (DTI) and volumetry. Cereb Cortex. 2010;20:2055-68. 